\title{
Análisis de variables físico-químicas en el proceso de remoción de coliformes en el sistema de lagunas de oxidación, Salguero, Valledupar (Colombia)
}

\author{
Pedro J. Fragoso-Castilla*; Luis A. Rubiano y José J. Kerguelen \\ Facultad de Ciencias de la Salud, Grupo de investigación Parasitología Agroecología Milenio, Univ. Popular del Cesar, \\ Valledupar-Colombia (correo-e: pedrofragozo@unicesar.edu.co; luisrubiano@unicesar.edu.co, \\ jjkerguelen@unicesar.edu.co)
}

${ }^{*}$ Autor a quien debe ser dirigida la correspondencia.

Recibido Jul. 13, 2020; Aceptado Sep. 10, 2020; Versión final Oct. 19, 2020, Publicado Feb. 2021

\begin{abstract}
Resumen
El objetivo de esta investigación fue analizar la relación entre variables físico-químicas y el porcentaje de remoción del grupo coliformes en el sistema de lagunas de oxidación de la ciudad de Valledupar, Colombia. Se recolectaron y analizaron 12 muestras de agua residual, cruda y tratada en temporada seca y de lluvia del sistema de tratamiento de aguas residuales Salguero en Valledupar. Las variables físico-químicas se midieron acorde al "Standard Methods for Examination of Water and Wastewater". La determinación del grupo coliformes se realizó mediante la técnica de filtración por membrana combinada con el método Merck de sustratos cromogénicos. Los resultados muestran una relación muy débil entre las variables físicas y el grupo coliformes. Se concluye que el sistema de tratamiento no cumple con las directrices nacionales e internacionales para el grupo coliformes, lo cual impacta negativamente sobre el rio Cesar y constituyen un problema de salud pública para las comunidades aledañas.
\end{abstract}

Palabras clave: coliformes termotolerantes; vertimientos; reutilización; aguas residuales; salud pública

\section{Analysis of physico-chemical variables during coliform removal from the Salguero oxidation lagoon system, Valledupar (Colombia)}

\begin{abstract}
The objective of this research study was to examine the relationship between physico-chemical variables and coliform removal percentage from the oxidation lagoon system in the city of Valledupar (Colombia). Twelve samples of wastewater, raw water, and treated water were collected during dry and rainy seasons from the Salguero wastewater treatment plant in Valledupar. Physico-chemical variables were measured according to the "Standard Methods for Examination of Water and Wastewater." Coliform group determination was performed by the membrane filtration technique and the chromogenic substrate Merck method. The results show weak relationships between physical variables and the coliform group. It is concluded that the treatment system does not comply with national and international guidelines for the coliform group. This has a negative impact on the Cesar River and constitutes a public health problem for surrounding communities.
\end{abstract}




\section{INTRODUCCIÓN}

El cambio climático ha intensificado la escasez de agua (Sabeen et al., 2019), la Organización de las Naciones Unidas para la Alimentación y la Agricultura (FAO), ha previsto que para el año 2050 se presentara un aumento de las temperaturas en todo el mundo entre un rango de $1,6^{\circ} \mathrm{C}$ a $6^{\circ} \mathrm{C}$ como máximo, como consecuencia, se verán disminuidas las fuentes de aguas superficiales (FAO, 2017); como alternativa para mitigar esta problemática, la Organización Mundial de la Salud (OMS) ha promovido la reutilización de aguas residuales como una estrategia para la reducción del gasto hídrico (OMS1, 2016). El uso de aguas residuales municipales parcialmente tratadas o no tratadas que se descargan al medio ambiente en un entorno agrícola, plantea riesgos para la salud humana, principalmente debido a la posible presencia de patógenos relacionados con excretas como son las bacterias del grupo coliformes (Zurita y Carreón, 2015). Existen diferentes estudios en la literatura que analizaron las características microbiológicas y fisicoquímicas de afluentes y efluentes para las evaluaciones de desempeño de los sistemas de tratamiento de aguas residuales (Adewumi y Ajibade, 2019; Ndulini et al., 2018 y Dhingra et al., 2015)

La contaminación de cuerpos de agua a causa de las descargas de aguas residuales, tiene tres aspectos de análisis; la concentración de microorganismos patógenos como los coliformes, que refleja el riesgo de infecciones; la calidad fisicoquímica del agua, en especial, el contenido de materia orgánica y finalmente los objetivos de calidad del agua en función de sus usos, donde se destaca la actividad agrícola y la preservación de la flora y fauna (Ugaz, 2018). A nivel mundial las aguas residuales son vertidas al medio ambiente sin un tratamiento adecuado, provocando contaminación en suelo, aguas superficiales y subterráneas (Abdalla y Khalil, 2018); Por lo tanto, es necesaria la implementación de proyectos de reutilización de agua y proyectos que apoyen la necesidad de realizar controles más eficientes en los vertimientos de los sistemas de tratamientos de aguas residuales STAR, (Maryam y Büyükgüngör, 2019; Unesco, 2017). La reutilización de aguas residuales se ve limitada por el funcionamiento adecuado de los STAR. En Colombia, cerca de 1.300 cuerpos de agua, están siendo contaminados por ser receptores de aguas residuales no tratadas 0 parcialmente tratadas, según reportes de la FAO (2016), contaba con alrededor de 410 sistemas de tratamiento de aguas residuales (STAR) con desconocimientos de los caudales vertidos a las fuentes receptoras. Solo un 33\% de los STAR construidos en el país tienen un adecuado funcionamiento.

Las aguas residuales son receptoras de una gran diversidad de microorganismos potencialmente transmisoras de enfermedades. Una de las formas de tratar las aguas residuales de origen doméstico, con el fin de disminuir su carga contaminante, para su posterior uso en la agricultura o en la industria en países en vías de desarrollo, es el empleo de las llamadas lagunas de oxidación o de estabilización. El tratamiento de las aguas residuales en la ciudad de Valledupar es realizado por un sistema de lagunas de oxidación estación denominado "El Salguero". De acuerdo con los informes de rendición de cuenta otorgada por la contraloría municipal de Valledupar, se presentan deficiencias en la operación y el mantenimiento; donde se destacan la presencia de gran cantidad de material flotante, falta de mantenimiento de los lechos de secado de lodos, falta de capacitación a los operarios y falta de análisis por laboratorio para el monitoreo de las variables físicoquímicas y microbiológicas necesarias para el monitoreo y control de la operación del sistema lagunar. Estas deficiencias provocan fallas en los procesos remoción microbiológica y fisicoquímica del STAR, lo que podría estar ocasionando vertimientos parcialmente tratados al rio Cesar, representando un riesgo para la población que habita y se abastece de este recurso aguas abajo del sitio de vertimiento.

Valledupar es de las pocas ciudades en el caribe colombiano, que trata el $100 \%$ de las aguas residuales. El sistema está constituido por tres fases. Un tratamiento primario consistente en desarenación y cribado. Una segunda fase consistente en lagunas anaeróbicas seguidas de facultativas y un tratamiento terciario consistente en lagunas de maduración (Núñez y Fragoso, 2020). En estos sistemas de tratamiento de aguas residuales los análisis físico-químicos y microbiológicos, son una parte importante en el diseño, mantenimiento y operación del sistema de lagunas. El objetivo de esta investigación analizar la relación existente entre las variables físico-químicas y el porcentaje de remoción de coliformes en el sistema de lagunas de oxidación, estación salguero en la ciudad de Valledupar - Colombia.

\section{MATERIALES Y MÉTODOS}

Se describe el área de estudio, la recolección de las muestras, la determinación del grupo coliformes, los análisis físicos y químicos realizados al agua residual y termina con el análisis de los datos.

\section{Área de estudio}

El sistema de lagunas de estabilización El Salguero (73¹4'07.15' O' y $\left.10^{\circ} 23^{\prime} 16.65^{\prime \prime} \mathrm{N}\right)$, se encuentra ubicado en el costado sur de la cabecera municipal, en inmediaciones de la llamada curva del Salguero, a unos 9 kilómetros del casco urbano de la Ciudad de Valledupar y a 116 m.s.n.m; con temperaturas máximas de 37º 
y un promedio $28^{\circ} \mathrm{C}$. la precipitación promedio fue de $1124 \mathrm{~mm}$. El sistema está constituida actualmente por una zona de cribado y desarenado, lagunas anaerobias, lagunas facultativas y de maduración (Figura 1).

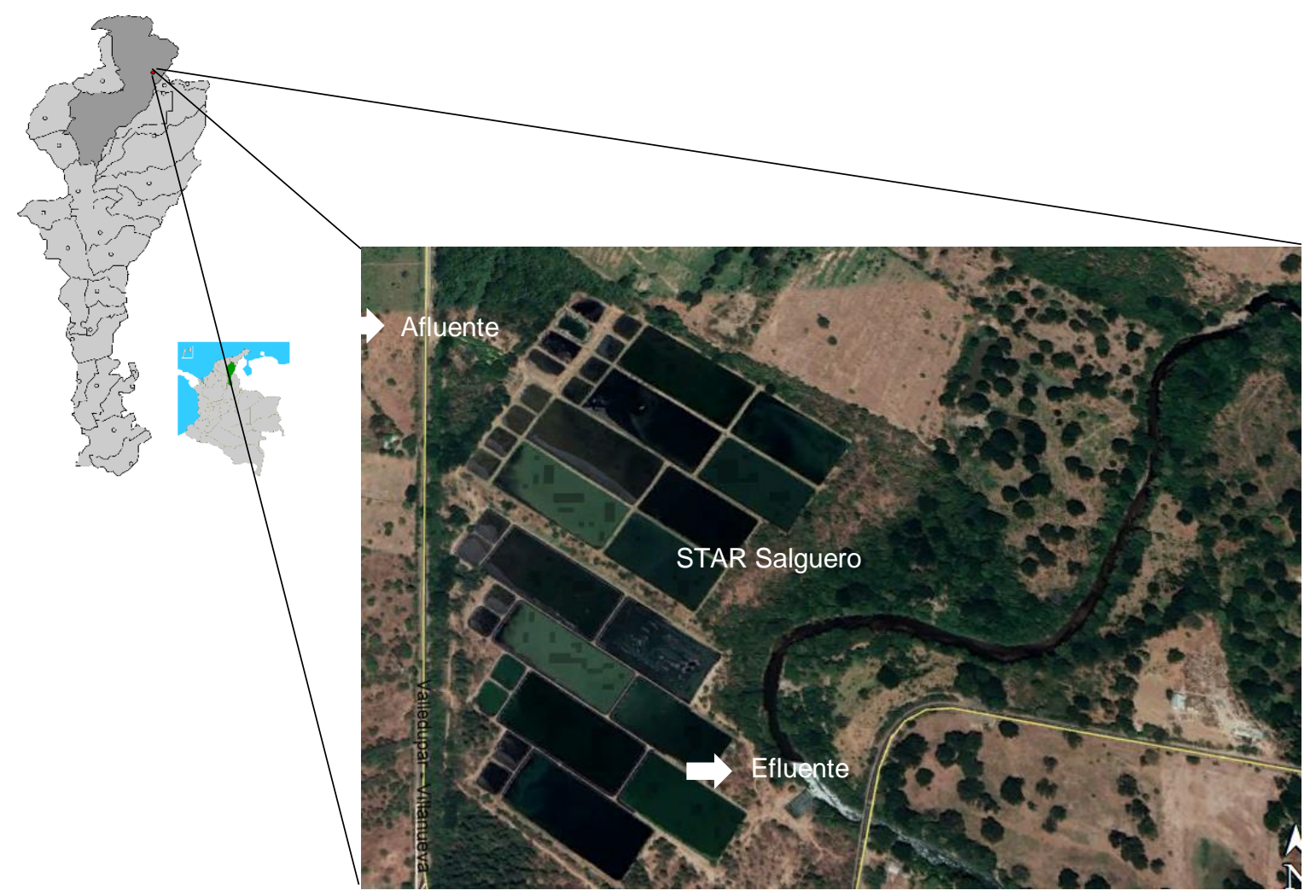

Fig. 1: Sistema de tratamiento de aguas residuales Salguero.

Las aguas residuales procedentes del sistema de alcantarillado de la ciudad de Valledupar, después de entrar a la planta, son conducidas hasta una estructura de entrada compuesta por cuatro módulos; cada uno de ellos consta de una estructura de cribado donde el agua pasa a través de un canal rectangular y allí atraviesa una rejilla metálica con una inclinación de 30, donde quedan retenidos los sólidos gruesos. De la zona de cribado, el agua pasa a la unidad de desarenado, compuesta por cuatro módulos, el agua procedente de los cuatro módulos de entrada, es conducida mediante tuberías de 27 " y pendiente de $0.1 \%$ a cuatro módulos, compuestos cada uno de ellos por dos lagunas anaeróbicas en paralelo, una laguna facultativa y una de maduración, para un total de 8 lagunas anaeróbicas, 4 facultativas y 4 de maduración., con profundidades de 4,2 y 1,5 metros respectivamente.

\section{Recolección de la muestra}

Se recolectaron un total de 12 muestras, seis (6) en temporada seca y seis (6) en temporada de lluvia, con intervalo entre muestras de 15 días, durante un periodo de seis meses; tomando como puntos de muestreo el canal de entrada del sistema de tratamiento y el canal de vertimiento. Para la evaluación del grupo coliformes se obtuvieron las muestras a $30 \mathrm{~cm}$ de profundidad en frascos de vidrio de $200 \mathrm{ml}$. Las muestras fueron rotuladas con la fecha, el $\mathrm{pH}$ y la turbiedad tomada durante el muestreo y conservadas a $4^{\circ} \mathrm{C}$ en una cava con pila refrigerante; Las muestras fueron transportadas al laboratorio Parasitología y Agroecología Milenio de la Universidad Popular del Cesar.

\section{Determinación de bacterias del grupo coliformes}

La contaminación por microorganismos patógenos en agua se determinó utilizando la cantidad de coliformes como indicador mediante la técnica de filtración por membrana en base al protocolo establecido por el Instituto de Hidrología, Meteorología y Estudios Ambientales (IDEAM, 2007). Las muestras de agua cruda fueron diluidas hasta $10^{-6}$; para el agua tratada se emplearon diluciones $10^{-5}$ y $10^{-6}$. Para la preparación de la dilución $10^{-6}$, primero se tomó $0.1 \mathrm{ml}$ de la muestra y se añadió en un frasco estéril con $99.9 \mathrm{ml}$ de agua destilada desionizada (dd) (dilución $10^{-3}$ ), posteriormente se tomaron $0,1 \mathrm{ml}$ de la dilución anterior y se diluyo en 99.9 $\mathrm{ml}$ de agua dd estéril (dilución $10^{-6}$ ). La dilución $10^{-5}$ se realizó tomando $1 \mathrm{ml}$ de agua y se añadió en $99 \mathrm{ml}$ de agua dd estéril (dilución $10^{-2}$ ), a partir de la dilución anterior se tomó $0.1 \mathrm{ml}$ diluyendo $99.9 \mathrm{ml}$ de agua dd estéril (dilución $10^{-5}$ ). Las muestras fueron procesadas en un equipo de filtración con bomba de vacío, utilizando membranas de $0,45 \mu \mathrm{m}$, las membranas fueron depositadas con pinzas estériles en cajas de Petri 
con agar Chromocult e incubadas a 37으 por 24-48 horas. Luego del periodo de incubación se seleccionaron las cajas con conteos inferiores a 200 UFC; Las colonias presuntivas de coliformes termotolerantes se confirmaron añadiendo una gota de reactivo de Kovac sobre la colonia, el viraje del reactivo confirmo la presencia de coliformes termotolerantes.

\section{Determinación de variables físicas y químicas}

Se determinaron las siguientes variables, de acuerdo con el protocolo establecido por (Rice y Baird, 2017): Parámetros químicos: pH (Método electrométrico 2550A) y conductividad (Método conductimétrico 2510A), Parámetros físicos: Solidos totales disueltos (TDS) (Método conductimétrico 2510A), Solidos totales sedimentables (SST) (Método gravimétrico 2540A), turbiedad (Método nefelométrico 2130A) y temperatura (Método electrométrico 2550A).

\section{Análisis de variables}

Se estimó la concentración de coliformes totales y termotolerantes y variables fisicoquímicas con base a la temporada seca y lluviosa y se establecieron las diferencias mediante un análisis de varianza de una vía (ANOVA) con el programa estadístico Statgraphics Centurión $\AA$, V 18, previa confirmación de los supuestos de normalidad y homocedasticidad. La correlación entre los parámetros fisicoquímicos y microbiológicos se realizó mediante un análisis de regresión lineal con el software StatPlus 6.5.0.0.

\section{RESULTADOS Y DISCUSIÓN}

Los resultados obtenidos a nivel de campo y laboratorio se presentan entre subsecciones: i). cuantificación de coliformes; ii) porcentaje de remoción de las variables analizadas y iii) Relación entre las variables físicas y químicas con la concentración de coliformes.

\section{Determinación de coliformes totales y termotolerantes en el STAR salguero en época seca y lluviosa}

El Ministerio de Ambiente y Desarrollo Sostenible (MADS) de la república de Colombia en el decreto 1076 de 2015, indica que para potabilizar el agua residual la fuente receptora debe contener una concentración máxima de 20.000 microorganismos $/ 100 \mathrm{ml}$ de coliformes totales. Los vertimientos del sistema de tratamiento se encuentran en valores superiores a lo establecido por las normas. En promedio para ambas temporadas el agua cruda presentó una concentración de $9 \times 10^{6} \mathrm{UFC} / 100 \mathrm{ml}$ y en el vertimiento $3 \times 10^{6} \mathrm{UFC} / 100 \mathrm{ml}$ (Tabla 1), para un porcentaje de remoción de $6,96 \%(p=0,0002)$ (Tabla 2) logrando una mayor remoción en la época seca.

La remoción de coliformes termotolerantes al igual que los totales, mantuvo una tendencia de mayor remoción

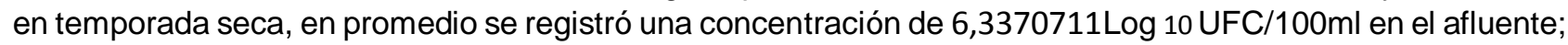
mientras que en el efluente se presentó una concentración promedio de 5,805292706 Log 10 UFC/100ml, la remoción general del sistema de tratamiento durante los días muestreados correspondió a $8,39 \%(p=$ 0,00005) (Tabla 2). La remoción general del sistema en durante los periodos muestreados corresponde a menos de una unidad logarítmica.

Tabla 1: Concentración de coliformes totales y termotolerantes en el STAR salguero en época seca y lluviosa

\begin{tabular}{|c|c|c|c|c|c|c|}
\hline \multirow[t]{2}{*}{ Temporada } & \multicolumn{3}{|c|}{ Coliformes totales UFC/100ml } & \multicolumn{2}{|c|}{$\begin{array}{c}\text { Coliformes } \\
\text { termotolerantes } \\
\text { UFC/100ml }\end{array}$} & \multirow[t]{2}{*}{ Valores de referencia } \\
\hline & № de Muestreo & Cruda & Tratada & Cruda & Tratada & \\
\hline \multirow{6}{*}{ Lluvia } & 1 & $6 \times 10^{6}$ & $4 \times 10^{6}$ & $4 \times 10^{6}$ & $2 \times 10^{6}$ & \multirow{12}{*}{$\begin{array}{l}\mathrm{FAO} \\
1 \times 10^{3} \mathrm{UFC} / 100 \mathrm{ml} \text { Coliformes } \\
\text { totales } \\
\text { Decreto } 1076 \text { De } 2015 \\
2 \times 10^{3} \text { microorganismos } / 100 \mathrm{ml} \\
\text { Coliformes termotolerantes }\end{array}$} \\
\hline & 2 & $26 \times 10^{6}$ & $3 \times 10^{6}$ & $1 \times 10^{6}$ & $1 \times 10^{5}$ & \\
\hline & 3 & $2 \times 10^{6}$ & $5 \times 10^{5}$ & $1 \times 10^{6}$ & $4 \times 10^{5}$ & \\
\hline & 4 & $14 \times 10^{6}$ & $11 \times 10^{6}$ & $3 \times 10^{6}$ & $2 \times 10^{6}$ & \\
\hline & 5 & $5 \times 10^{6}$ & $2 \times 10^{6}$ & $2 \times 10^{6}$ & $1 \times 10^{6}$ & \\
\hline & 6 & $9 \times 10^{6}$ & $7 \times 10^{6}$ & $3 \times 10^{6}$ & $1 \times 10^{6}$ & \\
\hline \multirow{6}{*}{ Seca } & 1 & $2 \times 10^{6}$ & $6 \times 10^{5}$ & $1 \times 10^{6}$ & $2 \times 10^{5}$ & \\
\hline & 2 & $13 \times 10^{6}$ & $6 \times 10^{6}$ & $7 \times 10^{6}$ & $3 \times 10^{6}$ & \\
\hline & 3 & $4 \times 10^{6}$ & $2 \times 10^{6}$ & $2 \times 10^{6}$ & $1 \times 10^{6}$ & \\
\hline & 4 & $13 \times 10^{6}$ & $11 \times 10^{5}$ & $5 \times 10^{6}$ & $4 \times 10^{5}$ & \\
\hline & 5 & $4 \times 10^{6}$ & $9 \times 10^{5}$ & $2 \times 10^{6}$ & $4 \times 10^{5}$ & \\
\hline & 6 & $10 \times 10^{6}$ & $3 \times 10^{6}$ & $11 \times 10^{5}$ & $3 \times 10^{5}$ & \\
\hline
\end{tabular}


Tabla 2: Remoción de parámetros físicos, químicos y microbiológicos del STAR el Salguero.

\begin{tabular}{|l|r|r|c|}
\hline Parámetros & Afluente & Efluente & \%Remoción \\
\hline Coliformes Totales (Log 10UFC/100ml) & $6,83 \pm 0,34$ & $6,36 \pm 0,42$ & 6,96 \\
\hline Coliformes Termotolerantes (Log 10UFC/100ml) & $6,33 \pm 0,29$ & $5,80 \pm 0,44$ & 8,39 \\
\hline Solidos Totales Disueltos g/ml & $335,58 \pm 74,77$ & $265,95 \pm 64,88$ & 20,74 \\
\hline Conductividad $\mu \mathrm{S}$ & $671,16 \pm 149,54$ & $531,91 \pm 129,77$ & 36,92 \\
\hline Solidos Sedimentables mI/L & $3,45 \pm 0,83$ & $0,40 \pm 0,12$ & 88,19 \\
\hline Turbiedad NTU & $152,73 \pm 75,10$ & $70,21 \pm 39,77$ & 54,02 \\
\hline pH & $7,22 \pm 0,14$ & $7,38 \pm 0,22$ & No aplica \\
\hline Temperatura ${ }^{\circ} \mathrm{C}$ & $23,62 \pm 1,38$ & $23,46 \pm 1,75$ & No aplica \\
\hline
\end{tabular}

De acuerdo con el Decreto 1076 de 2015, para la potabilización de las aguas residuales la concentración de coliformes termotolerantes de una fuente de abastecimiento debe ser inferior a 2000 Microorganismos/ 100ml, valor que no se cumple en el sistema de tratamiento durante el periodo de muestreo. Los coliformes son organismos que por su comportamiento siven como indicadores de microrganismos patógenos de allí su importancia como indicadores de calidad de los STAR especialmente si los vertimientos son reutilizados en actividades como el riego de cultivos (Verbyla et al; 2016). Campos et al. (2015) en un estudio similar determinaron la concentración de coliformes de aguas residuales tratadas utilizadas para el riego de cultivos de rábanos; los niveles de coliformes vertidos fueron inferiores a los registrados en el agua cruda. Sin embargo, no se encuentran completamente libre de contaminación por microorganismos patógenos para su utilización de riego de hortalizas, contrario a lo encontrado por Ayaz et al. (2015) los cuales evaluaron la calidad de un efluente y el potencial de reutilización de aguas residuales domésticas tratadas en un sistema de humedales, considerando una concentración inicial de coliformes totales y termotolerantes de $10^{7}$ coliformes / $100 \mathrm{ml}$, obteniendo a si un porcentaje de remoción de 99.00 y 99.99\%.

Al-Jassim et al., (2015), Evaluaron la eliminación de coliformes mediante procesos de lodo activado convencional de aguas residuales en Arabia Saudita; logrando una eliminación promedio logarítmica de $1.7 \pm$ 0.25 logs y $1.9 \pm 0.068$ logs para coliformes totales y termotolerantes respectivamente, cuyo afluente primario contenía una abundancia de coliformes termotolerantes que variaban de 105.2 a 106/ $100 \mathrm{ml}$; así mismo Osuolale y Okoh. (2015), Evaluaron las cualidades fisicoquímicas y la prevalencia de Escherichia coli en los efluentes finales de dos plantas de tratamiento de aguas residuales (PTAR), revelando la presencia de $E$. coli cuya concentración era de $1.86 \times 10^{4}$ UFC / $100 \mathrm{ml}$, llegando a la conclusión de que estas PTAR son fuentes importantes de contaminación en aguas superficiales con riesgos potenciales para la salud pública y ecológicos.

Los procesos de tratamiento realizados por el STAR salguero, disminuyeron la concentración de coliformes en el vertimiento, pero los valores absolutos obtenidos no permiten el cumplimiento de las normatividades vigentes, por consiguiente, se requiere la aplicación de alternativas de tratamientos que permitan la reducción de la concentración de coliformes en el vertimiento. Ndulini et al. (2018) Evaluaron la eliminación de coliformes termotolerantes en un sistema de tratamiento de aguas residuales, mediante la implementación de un sistema hidropónico, obteniendo una remoción del 92\%, llegando a la conclusión que la aplicación de este método pueden ofrecer una alternativa a los problemas del tratamiento en áreas donde los sistemas modernos no están disponibles o son ineficaces, así mismo, Emamjomeh et al. (2016) determinaron la eficiencia de tratamiento de aguas residuales sanitarias, basado en la combinación de sistemas anaerobios y de fitorremediación, logrando obtener altos porcentajes de remoción de coliformes totales.

\section{Determinación del porcentaje de remoción de las variables físicas, químicas y microbiológicas}

El Decreto 1076 de 2015 establece que para potabilizar aguas la turbiedad deberá ser menor o igual a 10 UJT (Unidades Jackson de Turbiedad) o su equivalente a 190 NTU, con base en este valor el sistema de tratamiento cumple con la normatividad.

El sistema de tratamiento en promedio removió un 54\% de la turbiedad del agua que ingreso (Tabla 2). Los valores máximos de turbiedad en el agua cruda se presentaron en temporada seca con un promedio de 191 NTU; mientras que para temporada de lluvia el valor promedio correspondió a 114 NTU $(p=0,001)$. Sin embargo, en temporada de lluvia se registraron valores de remoción de alrededor del $58,7 \%$ de la turbiedad, esto indica que el sistema de tratamiento a turbiedades bajas presenta una mejor eficiencia en el tratamiento. 
La determinación de los SST es de gran importancia en el tratamiento de las aguas residuales domésticas al permitir la determinación de la eficiencia de los procesos de sedimentación (Torres y Lozano, 2017); el sistema de tratamiento cumplió con el valor de SST establecidos en el Decreto 1076 de 2015, el cual estipula una concentración de solidos de $5 \mathrm{ml} / \mathrm{L}$. Los valores de SST en temporada de lluvia fueron mayores a la temporada seca en promedio en el agua cruda se registraron concentraciones de 3,66 ml/L de SST de los cuales se vertieron en promedio $0,43 \mathrm{ml} / \mathrm{L}$ obteniendo una remoción del $88,19 \%$ (Tabla 2)

Los sólidos disueltos representan el material soluble y coloidal, el cual requiere usualmente para su remoción, tratamiento anaeróbico, oxidación biológica o coagulación y sedimentación (UNESCO, 2017). La normatividad colombiana de ambiente no incluye los TDS como indicador de calidad de vertimientos, sin embargo, se ha empleado el valor referencial establecido por la FAO/ OMS, para vertimientos en aguas superficiales, en donde fija una TDS de 1000 mg/l (OMS2, 2006); con base en este valor la concentración de TDS del STAR salguero está bajo normatividad. El sistema de tratamiento presento valores máximos de TDS en agua cruda y tratada de $437 \mathrm{mg} / \mathrm{L}$ y $420 \mathrm{mg} / \mathrm{L}$ respectivamente $(p=0,01)$, estos datos se presentaron en temporada de verano (Tabla 2).

A pesar de estar bajo normatividad los porcentajes remoción de TDS fueron bajos; contrario a lo encontrado por Adewumi et al. (2019) los cuales realizaron una determinación periódica de las características fisicoquímicas y bacteriológicas de los efluentes de aguas residuales municipales generadas por la Universidad Federal de Tecnología de Akure, obteniendo como valor máximo (1408 mg/L). De manera general el sistema de tratamiento removió el 20,2\% de TDS que ingresaron al STAR; La temporada de lluvia presento una remoción de 22,43\% y la temporada seca registro una remoción del 18,12\% (Tabla 3).

La temperatura del agua residual influye en el desarrollo de la vida acuática, así como sobre las reacciones químicas y velocidades de reacción; se puede decir que a medida que aumenta la temperatura a aumenta la actividad de degradación de las bacterias (Tanyol y Demir, 2016), en el STAR Salguero, el agua cruda en promedio registro una temperatura de $23.6^{\circ} \mathrm{C}$ mientras que el agua tratada presento promedio de $23.5^{\circ} \mathrm{C}$ $(p=0.8)$, las temperaturas más altas se presentaron en temporada de lluvia. En el vertimiento se registraron temperaturas de alrededor de $24^{\circ} \mathrm{C}$ en temporada de lluvia y $23^{\circ} \mathrm{C}$ en temporada seca, en general el sistema de tratamiento cumplió con lo establecido en legislación Colombiana $\left(<40^{\circ} \mathrm{C}\right)$; valores diferentes a los resultados obtenidos por Dhingra et al. (2015), en un estudio sobre parámetros físico-químicos de efluentes de aguas residuales de áreas industriales de Jaipur, Rajasthan, India, cuyas temperaturas variaron de 29.4 ${ }^{\circ} \mathrm{C}$ a $34.8^{\circ} \mathrm{C}$, lo que puede llegar afectar la textura del suelo, si estas aguas se vierten directamente a la tierra y a su vez la proliferación de organismos la fuente receptora.

Los datos de $\mathrm{pH}$ del agua residual mostraron comportamientos ligeramente alcalinos en ambas temporadas, presentándose un aumento del $\mathrm{pH}$ vertido con relación al ingresado en la mayoría de los muestreos. El promedio de $\mathrm{pH}$ para el agua cruda fue de 7,22 mientras que para el agua tratada 7,39 $(p=0,02)$. El valor mínimo de $\mathrm{pH}$ en el agua cruda y tratada fue de 6,95 y 7,01 respectivamente valores presentados en la temporada lluviosa; mientras que los valores máximos registrados en temporada seca fueron de 7,43 y 7,80 para cruda y tratada; estos datos de $\mathrm{pH}$ en el vertimiento cumplen con el rango de $\mathrm{pH}$ estipulado en la normativa Colombiana vigente $(6,5-9,0)$

Los valores elevados de conductividad (>3000 $\mu \mathrm{S} / \mathrm{cm}$ ) afectan los procesos biológicos de depuración, dificultando el desarrollo de una comunidad bacteriana estable, provocando un desajuste en la colonia bacteriana, las bacterias filamentosas tienden a ser más estables lo que debilita la estructura flocular del lodo activo disminuyendo densidad y velocidad de sedimentación (Torres y Lozano, 2017). En temporada de lluvia el agua cruda registro una conductividad promedio de $611 \mu \mathrm{S} / \mathrm{cm}$ con un valor máximo de $823 \mu \mathrm{S} / \mathrm{cm}$ y un mínimo de $367 \mu \mathrm{S} / \mathrm{cm}$, mientras, que el vertimiento presento una conductividad promedio de $469 \mu \mathrm{S} / \mathrm{cm}$ $(p=0,08)$ con un valor máximo de $567 \mu \mathrm{S} / \mathrm{cm}$ y un mínimo de $288 \mu \mathrm{S} / \mathrm{cm}$ (Tabla 3). En temporada seca el promedio de conductividad fue mayor $(723 \mu \mathrm{S} / \mathrm{cm})$ con un valor máximo de $873 \mu S / \mathrm{cm}$ y un mínimo de 557 $\mu \mathrm{S} / \mathrm{cm}$ para el agua cruda; el agua tratada registro un valor máximo de $840 \mu \mathrm{S} / \mathrm{cm}$ y un mínimo de 493 $(p=0,06)$. La normatividad colombiana no incluye la conductividad como parámetro para calidad de vertimientos de los STAR, en países como España consideran la conductividad como un parámetro importante para indicar el efecto y calidad del vertimiento a fuentes receptoras; en este país en base al Decreto 109 de 2015 se establece que para vertimientos puntuales la conductividad debe ser menor a o igual a $2000 \mu \mathrm{S} / \mathrm{cm}$. Parámetro cumplido por el STAR Salguero.

\section{Relación entre las variables físicas y químicas con la concentración de coliformes.}

En las Fig 2 y 3 se detallan los resultados de la prueba de coeficiente de correlación de Pearson para cada una de las variables físicas y químicas con respecto a la concentración de coliformes totales y termotolerantes respectivamente. En cada uno de los casos se encontró que los valores no presentan una relación estadística significativa. 
En relación con las variables químicas analizadas se evidencia qué los coliformes logran un notable crecimiento en pH ligeramente alcalinos (entre 7 y 7,8) (Figura 2 y 3D) lo que puede atribuirse a los intensos procesos fotosintéticos, dada a la alta concentración de algas en el sistema, propios de las lagunas facultativa tal como lo comenta Matsumoto y Sánchez, (2016), mientras que los valores de conductividad estuvieron entre $400 \mu \mathrm{S}$ y $700 \mu \mathrm{S}$ (Figura 2 y $3 \mathrm{~A}$ ).

Valores significativamente diferentes a los encontrados en el estudio de Tanyol y Demir. (2016), Revelaron valores de conductividad de aguas residuales que variaron entre $1,055.75$ y $1,077.25 \mu S / \mathrm{cm}$ para el afluente y valores de efluente variadas entre $1,010.75$ y $1,067.02 \mu \mathrm{S} / \mathrm{cm}$, Sin embargo, dichos valor cumple con la normativa española. El valor de la conductividad refleja la cantidad de iones disueltos en el agua y se utiliza para determinar la idoneidad de agua para riego. Mediciones realizadas Osuolale y Okoh (2015), también informaron el cumplimiento de dos plantas de tratamiento de aguas residuales en Sudáfrica con respecto a estos mismos parámetros fisicoquímicos, como el pH, cuyos valores medidos 3.9-8.6. Sin embargo, durante todo el proceso presentaron relación directa con la alta presencia de coliformes en el efluente final.
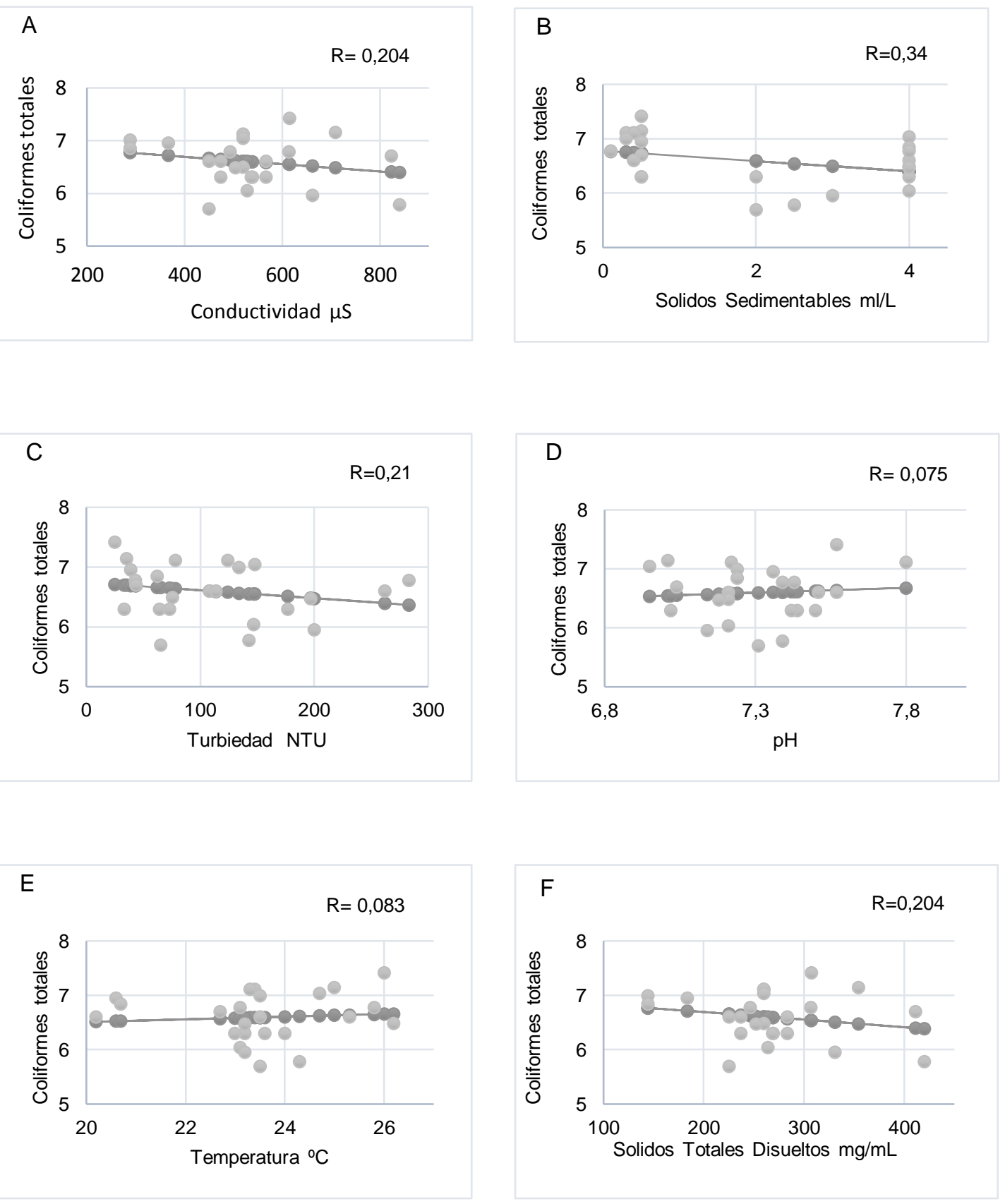

Fig. 2: Relación entre coliformes totales UFC Log10/100ml y (A) conductividad; (B) Sólidos sedimentables; (C) turbiedad; (D) $\mathrm{pH}$; (E) temperatura; (F) y solidos totales disueltos 

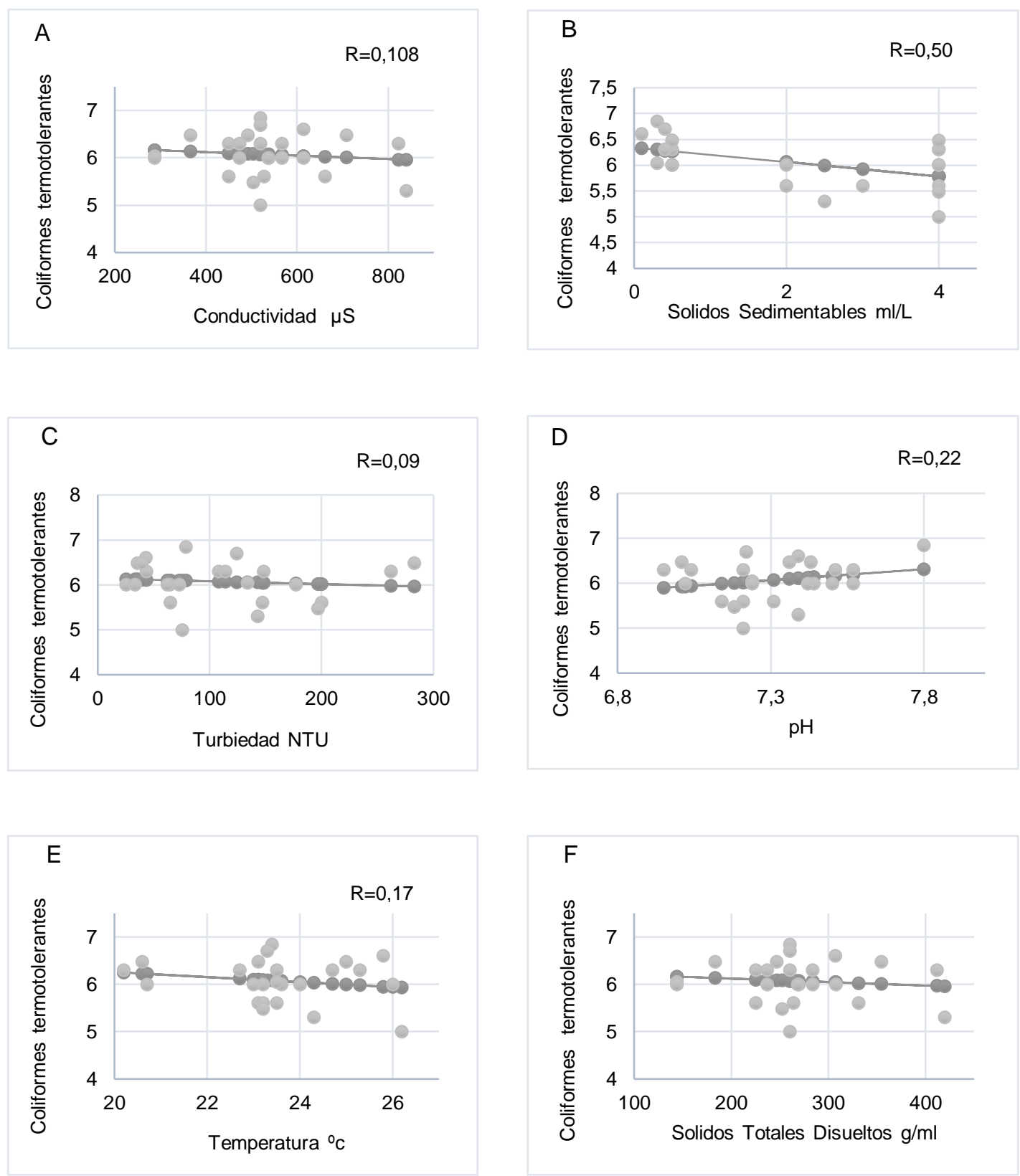

Fig. 3: Relación entre coliformes termotolerantes UFC Log10/100ml y (A) conductividad; (B) sólidos sedimentables; (C) turbiedad; (D) pH; (E) temperatura; (F) y solidos totales disueltos

El aumento o disminución de los niveles de turbiedad (Fig. 2 y 3 C) no presentaron efecto sobre la concentración de Coliformes, lo cual, contradice lo reportado por concordando con lo hallado por (Martinez et al; 2020) en donde en cuenta una relación fuerte entre los coliformes termotolerantes y la variable turbiedad, respaldado en el concepto de que la turbidez en el agua es causada principalmente por arena, limo, arcilla, fitoplancton, microorganismos o material orgánico (Kumar et al; 2015). Dentro de la investigación los sólidos sedimentables Fig. 2 y 3B, si bien, no presentaron relación estadística fuerte con el grupo coliformes totales, si mostraron relación mayor con la concentración de coliformes termotolerantes. El tratamiento biológico funciona en forma adecuada con un $\mathrm{pH}$ de 7; las variaciones en este parámetro pueden impactar significativamente en el correcto funcionamiento del sistema biológico de un STAR, incluso se puede llegar a inhibir la actividad microbiana, (Hernández et al; 2017).

El rango de TDS (Fig. 2 y 3 F) donde se encontró la mayor concentración de coliformes totales, estuvo comprendido entre $200 \mathrm{mg} / \mathrm{L}$ a $300 \mathrm{mg} / \mathrm{L}$. Lo anterior coincide con lo hallado por Osuolale y Okoh (2015), en donde las altas concentraciones de TDS registradas en dos plantas de tratamientos (Entre $253 \mathrm{mg} / \mathrm{L}$ y 336.3 $\mathrm{mg} / \mathrm{L}$ en la planta $1 ; 86.8 \mathrm{mg} / \mathrm{L}$ y $127.5 \mathrm{mg} / \mathrm{L}$ en la planta 2) conllevaron a una mayor remoción de $E$ coli (En promedio, $1.86 \times 10^{4} \mathrm{UFC} / 100 \mathrm{ml}$ ). Las temperaturas (Fig. 2 y $3 \mathrm{E}$ ) en las cuales se encontró una mayor abundancia de coliformes estuvieron comprendidas entre $23^{\circ} \mathrm{C}$ y $26^{\circ} \mathrm{C}$. Cabe resaltar que las altas 
temperaturas pueden atribuirse a la retención prolongada en los estanques de oxidación, lo que genera ablandamiento de las articulaciones bituminosas y a su vez aumenta el olor generado por la reacción anaeróbica (Agoro et al; 2018).

\section{CONCLUSIONES}

El análisis efectuado a nivel de campo y laboratorio permite concluir lo siguiente: 1. Los valores absolutos y porcentajes de remoción de coliformes totales y termotolerantes del STAR Salguero no cumplen con las normativas internacionales y nacionales vigentes para vertimientos de aguas en fuentes receptoras. 2. El STAR salguero requiere de un sistema de tratamiento adicional, que contribuyan a la remoción de coliformes. 3. No existe una correlación entre las variables físicas y la remoción de coliformes, lo cual impide utilizarlas como medida indirecta del proceso de remoción microbiológica de las lagunas de oxidación. 4. El vertimiento de las aguas residuales del STAR salguero al rio Cesar se convierten en un problema de salud pública para las poblaciones aledañas.

\section{AGRADECIMIENTOS}

Los autores reconocen la ayuda del programa de Microbiologia de la Universidad Popular del Cesar, Colombia y el apoyo logistico realizado por La Empresa de Servicios Públicos de Valledupar - Cesar. EMDUPAR S.A. ESP.

\section{REFERENCIAS}

Abdalla, F y Khalil, R., Potential effects of groundwater and surface water contamination in an urban area, Qus City, Upper Egypt. https://doi.org/10.1016/j.jafrearsci.2018.02.016, Journal of African Earth Sciences, 141, 164-178 (2018)

Adewumi, J.R., y Ajibade, F.O., Periodic determination of physicochemical and bacteriological characteristics of wastewater effluents for possible reuse as irrigation wáter, https://doi.org/10.1007/s42108-019-00036-6, International Journal of Energy and Water Resources ,3(4), 269-276 (2019).

Agoro, M. A., Okho, O. O., y otros dos autores, Physicochemical properties of wastewater in three typical South African sewage Works, https://doi.org/10.15244/pjoes/74156, Polish Journal of Environmental Studies, 27(2), 491-499 (2018).

Al-Jassim, N., Ansari, M. I., y otros dos autores, Removal of bacterial contaminants and antibiotic resistance genes by conventional wastewater treatment processes in Saudi Arabia: is the treated wastewater safe to reuse for agricultural irrigation, https://doi.org/10.1016/j.watres.2015.01.036, Water research ,73, 277-290 (2015).

Ayaz, S, Ç., Artas, O., y otros dos autores, Effluent quality and reuse potential of domestic wastewater treated in a pilotscale hybrid constructed wetland system, https://doi.org/10.1016/j.jenvman.2015.03.042, Journal of environmental management, 156, 115-120 (2015).

Campos, C., Contreras, A. M., y Leiva, F. R., Evaluación del riesgo sanitario en un cultivo de lechuga (lactuca sativa) debido al riego con aguas residuales sin tratar en el Centro Agropecuario Marengo (Cundinamarca, Colombia), http://dx.doi.org/10.17151/biosa.2015.14.1.8, Biosalud, 14 (1), 69-79 (2015).

Dhingra, P., Singh, Y., y otros cuatro autores, Study on physicochemical parameters of waste water effluents from industrial areas of Jaipur, Rajasthan, India. Int. J. Innov. Sci. Eng. Technol, 2(5), 874-876 (2015).

Emamjomeh, M. M., Hamaly H. A., y otros tres autores, Sanitary wastewater treatment using combined anaerobic and phytoremediation systems. Journal of Mazandaran University of Medical Sciences 26.138: 140-150 (2016).

FAO; Colombia uso del agua. Organización de las naciones unidas para la alimentación y la agricultura (2016).

FAO; Escasez de agua: Uno de los grandes retos de nuestro tiempo. Organización de las Naciones Unidas para la Alimentación y la Agricultura. (2017).

García, H. A., Buitrón, G., y otros dos autores, Metabolismo Microbiano, Tratamiento biológico de aguas residuales: principios, modelación y diseño, pp 29-30, IWA Publishing (2017).

IDEAM; Determinación De Escherichia Coli y Coliformes Totales en agua por el método de filtración por membrana en agar Chromocult. Instituto de Hidrología, Meteorología y Estudios Ambientales. República de Colombia (2007).

Martínez-Orjuela, M., Mendoza-Coronado, J., y otros tres autores. Evaluación de la turbiedad como parámetro indicador del tratamiento en una planta potabilizadora municipal. https://doi.org/10.18273/revuin.v18n4-2020001, Revista UIS Ingenierías, 19(1), 15-24 (2020)

Maryam, B., y Büyükgüngör, H., Wastewater reclamation and reuse trends in Turkey: Opportunities and challenges, https://doi.org/10.1016/j.jwpe.2017.10.001. Journal of Water Process Engineering, 30, 100501 (2019)

Matsumoto, T., y Sánchez-Ortiz, I. A., Desempeño de las lagunas anaerobia con bafle divisor y facultativa de la PTAR de Santa Fe do Sul (São Paulo, Brasil). http://dx.doi.org/10.22395/rium.v15n29a2, Revista Ingenierías Universidad de Medellín, 15(29), 27-42 (2016). 
Ndulini, S. F., Sithole, G. M., y Mthembu, M. S., Investigation of nutrients and faecal coliforms removal in wastewater using a hydroponic system, https://doi.org/10.1016/j.pce.2018.05.004, Physics and Chemistry of the Earth, Parts A/B/C, 106, 6872 (2018).

Núñez, J. C., y Fragoso-Castilla, P. J., Uso de macroinvertebrados acuáticos como bioindicadores de contaminación del agua de la Ciénaga Mata de Palma, Colombia, http://dx.doi.org/10.4067/S0718-07642019000500319, Revista Información Tecnológica 30 (5): 319-330 (2019)

OMS1; Escases de agua. Organización Mundial de la Salud. Datos y Cifras, 10pp. (2016).

OMS2; Guías para la calidad del agua potable, Organización Mundial de la Salud. 189pp (2006).

Osuolale, O., y Okoh, A., Assessment of the physicochemical qualities and prevalence of Escherichia coli and vibrios in the final effluents of two wastewater treatment plants in South Africa: Ecological and public health implications, https://doi.org/10.3390/ijerph121013399, International journal of environmental research and public health 12(10) 1339913412 (2015).

Rice E.W., y Baird, B., Standard Methods For the examination of water and wastewater. Asociación Americana de Salud Pública, Asociación Americana de Obras Hidráulicas, Federación de Medio Ambiente del Agua, ISBN: 9780875532875. (2017).

Sabeen, A. H., Kamaruddin, S. N., y Noor, Z. Z., Environmental impacts assessment of industrial wastewater treatment system using electroless nickel plating and life cycle assessment approaches, https://doi.org/10.1007/s13762-018-19746, International journal of environmental science and technology, 16(7), 3171-3182 (2019).

Tanyol, M., y Demir, V., Correlations between some operation parameters and efficiency evaluation of domestic wastewater treatment plant in Tunceli (Turkey), https://doi.org/10.1080/19443994.2016.1182082, Desalination and Water Treatment 57(58) 28115-28121 (2016).

Torres, G. A., y Lozano, E. A., Disminución de sólidos de aguas grises mediante un proceso de aireación. Ra Ximhai, ISSN: 1665-0441, 13(3), 393-404, (2017).

Ugaz, F. E., Reúso de aguas residuales tratadas biológicamente, para el regadío del Jardín Botánico, Trujillo, http://dx.doi.org/10.17268/sciendo.2018.001, SCIÉNDO 21(1) 7-14 (2018).

UNESCO; Aguas Residuales El Recurso Desaprovechado. Organización de las Naciones Unidas para la Educación, la Ciencia y la Cultura. Informe. Paris, Francia; pp 180 (2017).

Verbyla, M. E., Iriarte, M., y otros cuatro autores, Pathogens and fecal indicators in waste stabilization pond systems with direct reuse for irrigation: Fate and transport in water, soil and crops, https://doi.org/10.1016/j.scitotenv.2016.01.159, Science of the Total Environment 551: 429-437 (2016).

Yadav, K. K., Gupta, N., y otros tres autores, Water quality assessment of Pahuj River using water quality index at Unnao Balaji, MP, India. Int J Sci Basic Appl Res, ISSN 2307-4531, 19(1), 241-250 (2015).

Zurita, F., y Carreón-Álvarez, A., Performance of three pilot-scale hybrid constructed wetlands for total coliforms and Escherichia coli removal from primary effluent-a 2-year study in a subtropical climate, https://doi.org/10.2166/wh.2014.135, Journal of water and health ,13(2), 446-458 (2015). 